\title{
A Novel Approach to Enhancement Linked Laser Asymmetric Keratectomy Using Semi-Cylindrical Ablation Pattern in Patients with Myopic Regression After Laser Refractive Surgery
}

\author{
Ji Sang Min' \\ Byung Moo Min ${ }^{2}$ \\ 'Kim's Eye Hospital, Division of Cornea, \\ Cataract, and Refractive Surgery, \\ Konyang University School of Medicine, \\ Seoul, South Korea; ${ }^{2}$ Woori Eye Clinic, \\ Department of Ophthalmology, Yonsei \\ University School of Medicine, Daejon, \\ South Korea
}

Purpose: We aimed to introduce a new technique to reduce regional asymmetry of corneal thickness by assessing its effectiveness in four patients with myopic regression after laser refractive surgery (LRS).

Patients and Methods: Four patients (four eyes) with myopic regression after LRS were included in this study. A new technique of enhancement with laser epithelial keratomileusislinked laser asymmetric keratectomy using semi-cylindrical ablation pattern (E-LAK-SCAP) with full integration of the Vision-Up software for analyzing the corneal thickness deviation can be used to create central symmetry by blocking laser ablation on the thin cornea. It reduces the regional asymmetry of the corneal thickness, thus improving corneal symmetry and correcting the refractive power and myopic shift due to E-LAK-SCAP. We measured refraction, visual acuity, intraocular pressure (IOP), central corneal thickness (CCT), corneal irregularities in the $3.0 \mathrm{~mm}$, and 5.0 zones on Orbscan maps, the sum of corneal thickness deviations in four directions (SUM), distance between the maximum posterior elevation (best-fit-sphere [BFS]) and the visual axis (DISTANCE), and angle kappa before and after LRS and E-LAK-SCAP. Blurring scores were measured before and after E-LAK-SCAP.

Results: The uncorrected far visual acuity (LogMAR) increased after LRS and E-LAKSCAP. SUM $(\mu \mathrm{m})$ increased after LRS in three cases, but decreased in all four cases after E-LAK-SCAP. DISTANCE increased after LRS, but decreased after E-LAK-SCAP. The spherical equivalent, CCT, decreased after LRS and E-LAK-SCAP. Blurring scores decreased after E-LAK-SCAP, and angle kappa was similar before and after LRS, but decreased after E-LAK-SCAP. IOP was similar before and after both LRS and E-LAKSCAP.

Conclusion: E-LAK-SCAP improved corneal symmetry by reducing the SUM and DISTANCE, showing good postoperative visual acuity, and blurring was reduced postoperatively. There was no myopic regression in the one-year postoperative period.

Keywords: regional asymmetry of corneal thickness, myopic regression, LRS, E-LAKSCAP

Correspondence: Byung Moo Min Woori Eye Clinic, Department of Ophthalmology, Yonsei University School of Medicine, Daeduk Daero 219 (Dunsandong), Seo-gu, Daejon, 35229, South Korea

Tel +82-42-476-1675

Fax +82-42-476-1670

Email bmin8275@naver.com

\section{Introduction}

If a large deviation in corneal thickness is observed after laser refractive surgery (LRS) of the eye, the thin regions of the cornea are more likely to be steepened as a result of intraocular pressure, leading to increased changes in myopic regression. 
Therefore, it is very important to reduce regional asymmetry of corneal thickness in LRS. ${ }^{1-5}$ If only enhancement with laser-assisted in-situ keratomileusis (LASIK) or laser assisted sub-epithelial keratectomy (LASEK) was performed in patients with myopic regression with a sum of deviations in corneal thickness in four directions (SUM) of $\geq 80 \mu \mathrm{m}$, postoperative myopic regression could reoccur. ${ }^{3}$ Therefore, enhancement with reduced regional asymmetry of corneal thickness could be one of the options for improved treatment.

Recently, laser asymmetric keratectomy (LAK) has been reported to reduce regional asymmetry of corneal thickness. Enhancement linked laser symmetric keratectomy using semi-cylindrical ablation pattern (E-LAKSCAP) is a customized asymmetric corneal ablation method with full integration of Vision-Up software, and a new device to analyze corneal thickness deviation, ${ }^{1-3}$ correct refractive errors and also reduce the total corneal thickness deviation in central symmetry. It has shown excellent surgical results, including reduced blurring scores and no myopic regression, with a SUM of $\geq 80$ $\mu \mathrm{m}$ one-year postoperatively. ${ }^{2,3}$ Additionally, one-partial ablation is possible in the cornea using the existing cylindrical ablation pattern to reduce regional asymmetry of corneal thickness.

We present a new technique of E-LAK-SCAP with full integration of Vision-Up software to correct refractive errors and reduce regional asymmetry of corneal thickness in patients with myopic regression after LRS.

\section{Patients and Methods}

This retrospective study included patients who were treated at the Woori Eye Clinic between February 2018 and October 2018. Four patients (4 eyes) who underwent L-LAK-SCAP, and who showed myopia of more than -1.0 diopter, a SUM of $\geq 80 \mu \mathrm{m}$ after LRS, and followup check over one year were included in this study. Patients with follow-up examination under one year, and history of other ocular operations or trauma were excluded. The pre- and post-LRS and pre-and one-year post-E-LAK-SCAP results were retrospectively analyzed. This study was conducted in accordance with the Helsinki Declaration of 1975, as revised in 1983, and approved by the Korean National Institute for Bioethics Policy. Written informed consent was obtained from all the patients.

Patients with refractive errors underwent E-LAK-SCAP using a 193-nm ISO-D 200 laser (Kera Harvest Inc., Taiwan). Laser correction was performed by the same surgeon (BM
Min) using an identical method for all patients. Local anesthesia was induced by instillation of $0.50 \%$ proparacaine hydrochloride (Alcaine, Alcon NV, Vilvoorde, Belgium). For LASEK, a 9.0-9.5-mm diameter patch of corneal epithelium was removed with a brush. For refractive correction, laser ablation was performed in the $6.0-6.5 \mathrm{~mm}$ optic zone to correct myopia and astigmatism. To perform LAKSCAP, ${ }^{1-3}$ we used the Vision-Up software (WellC, South Korea) to analyze the corneal deviations based on Orbscan II (Bausch \& Lomb, Bridgewater, NJ, USA) corneal maps. These also served as predictors of corneal myopic change as a result of the removal of the thicker corneal regions, as determined by LAK-SCAP. Therefore, we were able to ablate the cornea to create central symmetry without changing the refractive power. ${ }^{1-3} \mathrm{~A}$ rating $^{6}$ for blurring was recorded under interview with glasses or contact lens correction using a subjective scale: $0=$ none, $1=$ mild, $2=$ moderate, and 3 = severe or disturbing, before and after E-LAKSCAP. The following variables were analyzed: the pre- and postoperative (for both LRS and E-LAK-SCAP) uncorrected distance visual acuity (UDVA), spherical equivalent (SE), sphere, cylinder, intraocular pressure (IOP), kappa angle on Orbscan map, central corneal thickness (CCT), corneal irregularities in the 3.0 -and $5.0 \mathrm{~mm}$ zones on Orbscan maps, pupil size, SUM $(\mu \mathrm{m})$, and distance between the maximum posterior elevation (best-fit-sphere [BFS]) and the visual axis (DISTANCE; mm). Blurring severity was checked before and after E-LAK-SCAP. The refraction was measured using an auto refractometer/keratometer and was calculated as the SE.

UDVA was measured at a distance of $3 \mathrm{~m}$ using the Han Chun Suk visual distance acuity chart, and was converted to the logarithm of the minimum angle of resolution (LogMAR). Myopic regression during the follow-up period was defined as changes in myopia, indicated by a spherical equivalent (SE) $\geq 1.0 \mathrm{D}$ after the surgery.

Pre- and postoperative deviations in corneal thickness (asymmetric pachymetric distribution) were analyzed using the following method: first, the thickness was measured using Orbscan maps in 8 locations $\left(0^{\circ}, 45^{\circ}, 90^{\circ}, 135^{\circ}\right.$, $180^{\circ}, 225^{\circ}, 270^{\circ}$, and $\left.315^{\circ}\right), 2.5 \mathrm{~mm}$ from the center of the cornea; the differences in thickness between symmetrically opposed locations were then calculated for four directions $\left(0-180^{\circ}, 45-225^{\circ}, 90-270^{\circ}\right.$, and $\left.135-315^{\circ}\right)$, along with the sum of the differences (Figures 1-3). ${ }^{1-3}$ DISTANCE was analyzed by converting the distance between the $\mathrm{X}$ and $\mathrm{Y}$ coordinates of the thinnest point and the center of the cornea on an Orbscan map (Figures 1-3). ${ }^{1-3}$ 


\section{DORBSCAN}

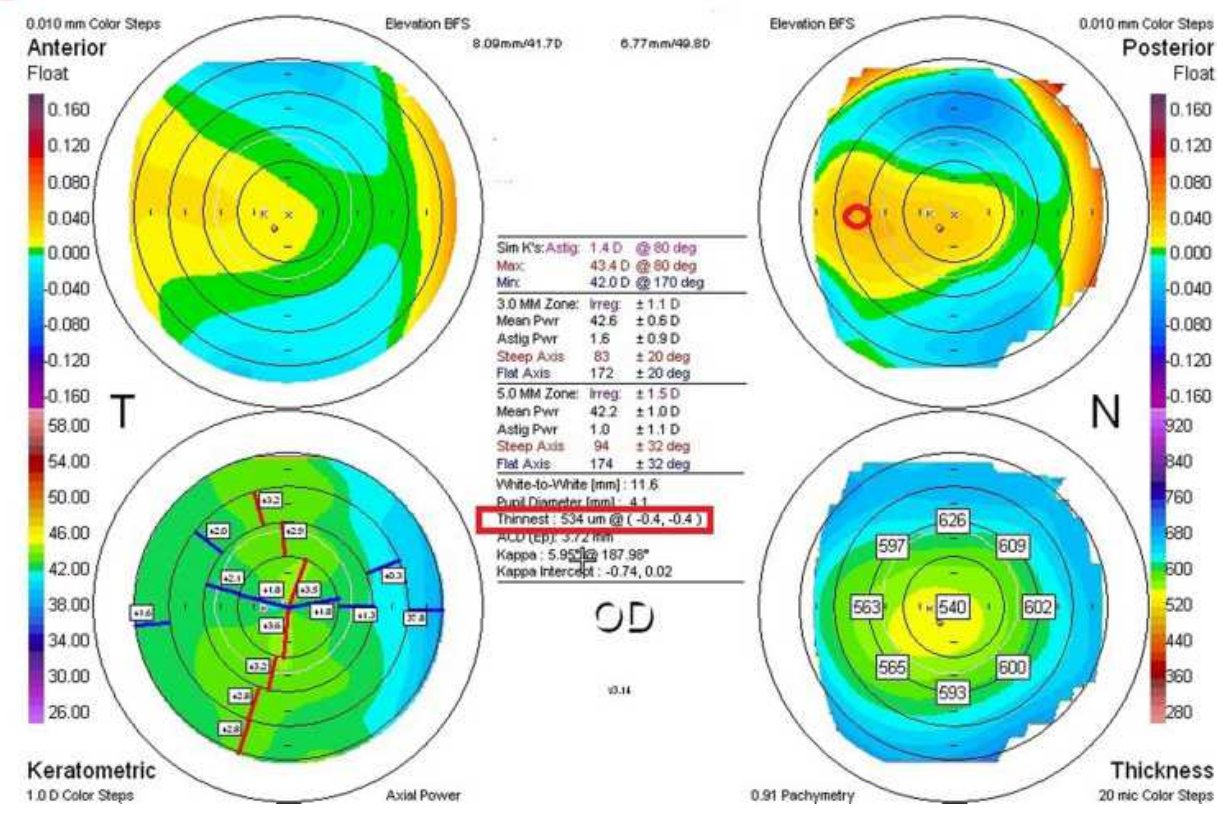

Figure I Pre-LRS Orbscan map of case I. Right bottom shows a pachymetric map: an example of measuring the differences in thickness between symmetrically opposed

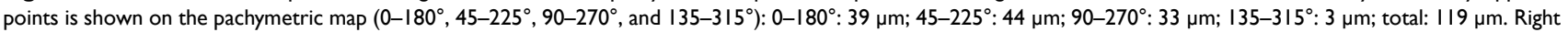
top map: Measuring the distance between the maximum posterior elevation (best-fit-sphere; BFS) and the visual axis. Corneal apex: temporally deviated (right upper red circle). The thinnest point $(X, Y)$ is indicated by the lower red square, and the posterior high on thicker cornea, and front elevation on the thinner cornea is also shown.

\section{DORBSCAN}

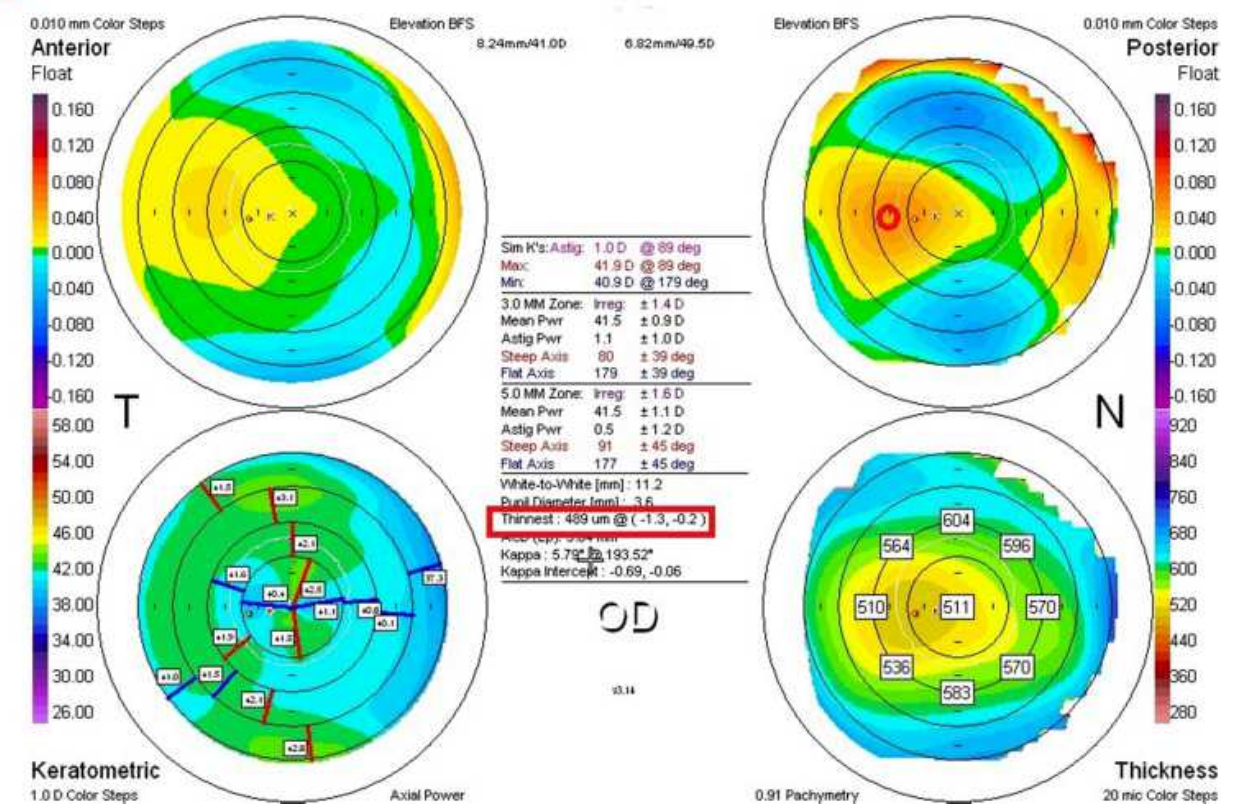

Figure 2 Pre-E-LAK-SCAP (post-LRS) Orbscan map of case I. Right bottom pachymetric map: an example of measuring the differences in thickness between symmetrically opposed points is shown on the pachymetric map $\left(0-180^{\circ}, 45-225^{\circ}, 90-270^{\circ}\right.$, and $\left.135-315^{\circ}\right)$ : 0-180 : $60 \mu \mathrm{m} ; 45-225^{\circ}: 60 \mu \mathrm{m} ; 90-270^{\circ}: 21 \mu \mathrm{m} ; 135-315^{\circ}: 6 \mu \mathrm{m} ;$ total: 147 $\mu \mathrm{m}$. Right top map: shows the measured distance between the maximum posterior elevation (best-fit-sphere; BFS) and the visual axis. Corneal apex: temporally deviated (right upper red circle). The thinnest point $(X, Y)$ is indicated by the lower red square. 


\section{Surgical Technique of E-LAK-SCAP}

To perform E-LAK-SCAP, ${ }^{1-3}$ we used Vision Up software (WellC, South Korea) to analyze the corneal thickness deviations based on Orbscan II (Bausch \& Lomb, Bridgewater, NJ, USA) corneal maps (Figures 1-3), to predict the amounts of ablation on a thicker corneal region, and to determine the direction of the axis (degree), number of SCAPs, and corneal myopic change as a result of removal of the thicker corneal regions as determined by LAK-SCAP (Figure 4). Figure 4 illustrates how LAK can

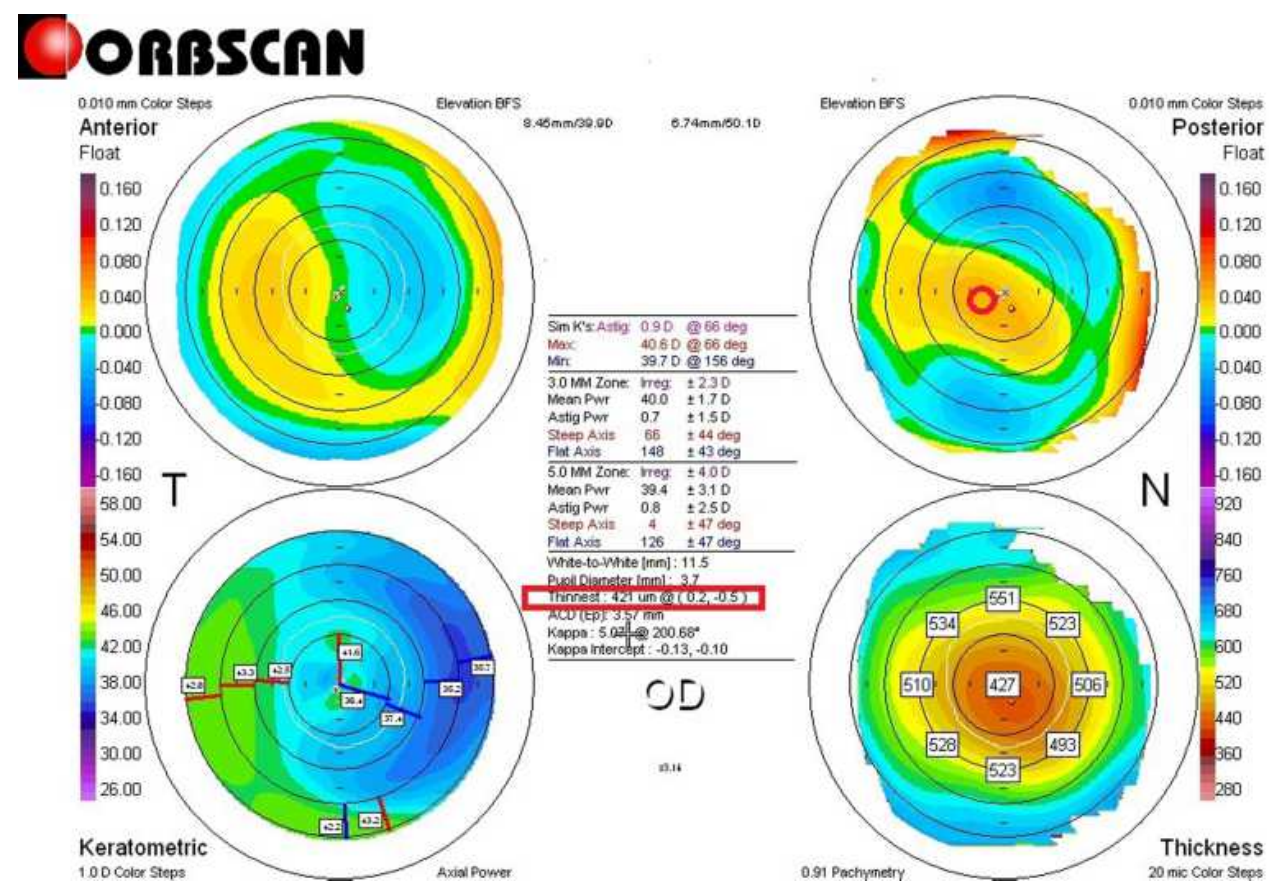

Figure 3 Post-E-LAK-SCAP Orbscan map of case I. Right bottom pachymetric map: an example of measuring the differences in thickness between symmetrically opposed

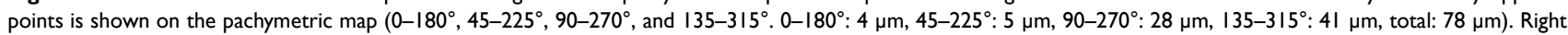
upper map: the measured distance between the maximum posterior elevation (best-fit-sphere; BFS) and the visual axis is also shown. Corneal apex: centrally located (right upper red circle). The thinnest point $(X, Y)$ is indicated by the lower red square.

A

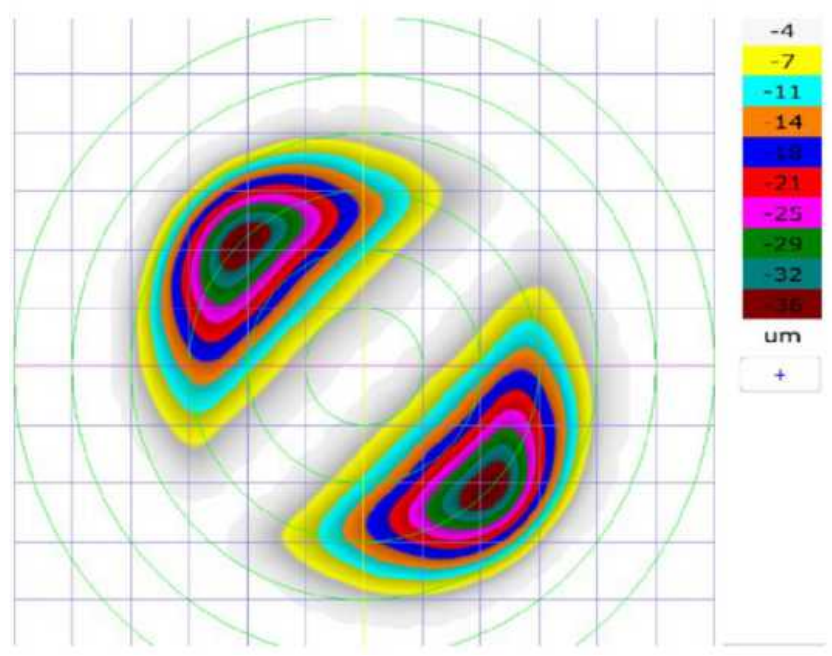

B

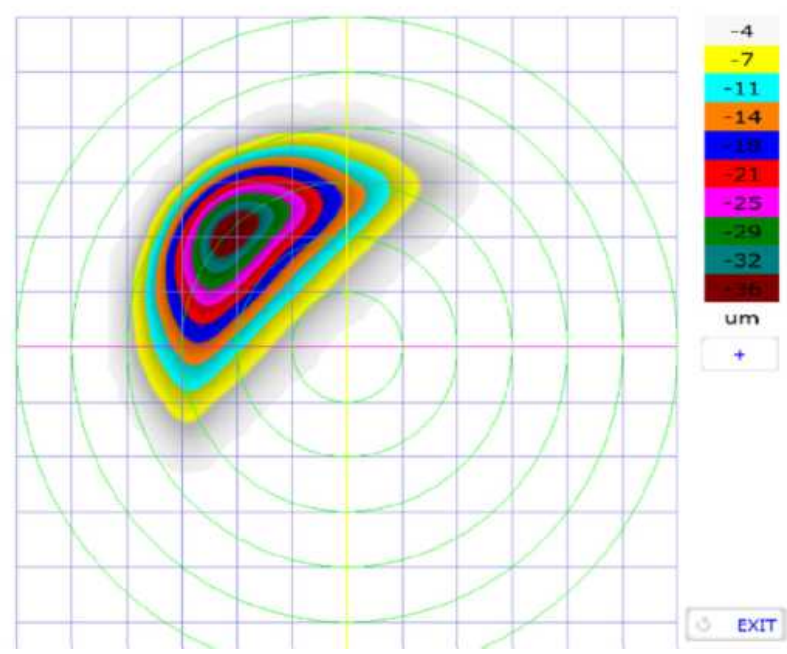

Figure 4 The schematic description of LAK-SCAP. (A) shows examples of axial, symmetric images of two-partial ablation patterns. (B) shows examples of laser ablation patterns for LAK-SCAP; specifically, it shows one partial ablation for existing cylindrical ablation patterns, as seen in (A). These patterns can be achieved by blocking laser corneal ablations at the thinner sections of the cornea. 
be implemented with a cylindrical ablation pattern for conventional astigmatism correction. Figure $4 \mathrm{~A}$ shows examples of axial and symmetric images of the twopartial ablation patterns; Figure 4B shows examples of one-partial (semi-cylindrical) laser ablation pattern for LAK-SCAP. These patterns can be achieved by blocking laser corneal ablations in the thinner sections of the cornea. E-LAK-SCAP improved corneal symmetry by reducing the sum of corneal thickness deviations, and correcting refractive power and myopic shift due to LAKSCAP, simultaneously (Figures 5 and 6). Therefore, we were able to ablate the cornea to create central symmetry, without changing refractive power (Figure 3 ). ${ }^{1-3}$

\section{Results}

The age range of the patients was 19-46 years (average: 28.5 years) for LRS, and 20-48 years for E-LAK-SCAP (average: 32.8 years). The male-to-female ratio was $1: 1$, interval between LRS and E-LAK-SCAP was 8-132 months (average: 58.3 months) (Table 1). Intraoperatively, optic zone $(\mathrm{mm})$ in LRS and LAK-SCAP was 6.0-6.2, ablation depth $(\mu \mathrm{m})$ was 48-90 (average: 70) for LRS, and 30-75 (average:50) for E-LAK-SCAP, myopic shift (diopters) due to LAK-SCAP was $-1.50--2.50$ (average: 2.0 ), residual

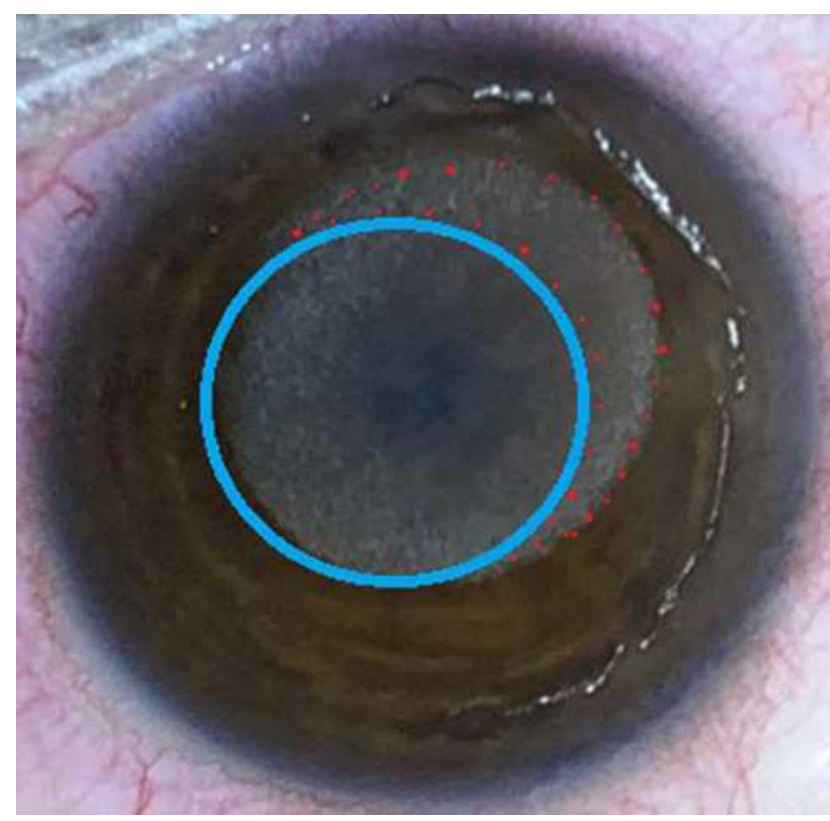

Figure 5 Ablation pattern of case I: Purple circled round area indicates ablation pattern (total $=5.50$ diopters) of both refractive error ( -4.0 diopters) and myopic shift ( -1.50 diopters) due to LAK-SCAP, and red dotted circled area indicates LAKSCAP on the thicker area of the cornea.

stromal depth $(\mu \mathrm{m})$ was 391-420 (average: 404$)$ for LRS, and 321-391 (average: 366) at E-LAK-SCAP (Table 2).

For pre and postoperative findings of two operations (Table 3), SE (diopters) were $-3.38--7.50$ (average: -5.47 ) before

\section{DORBSCAN}

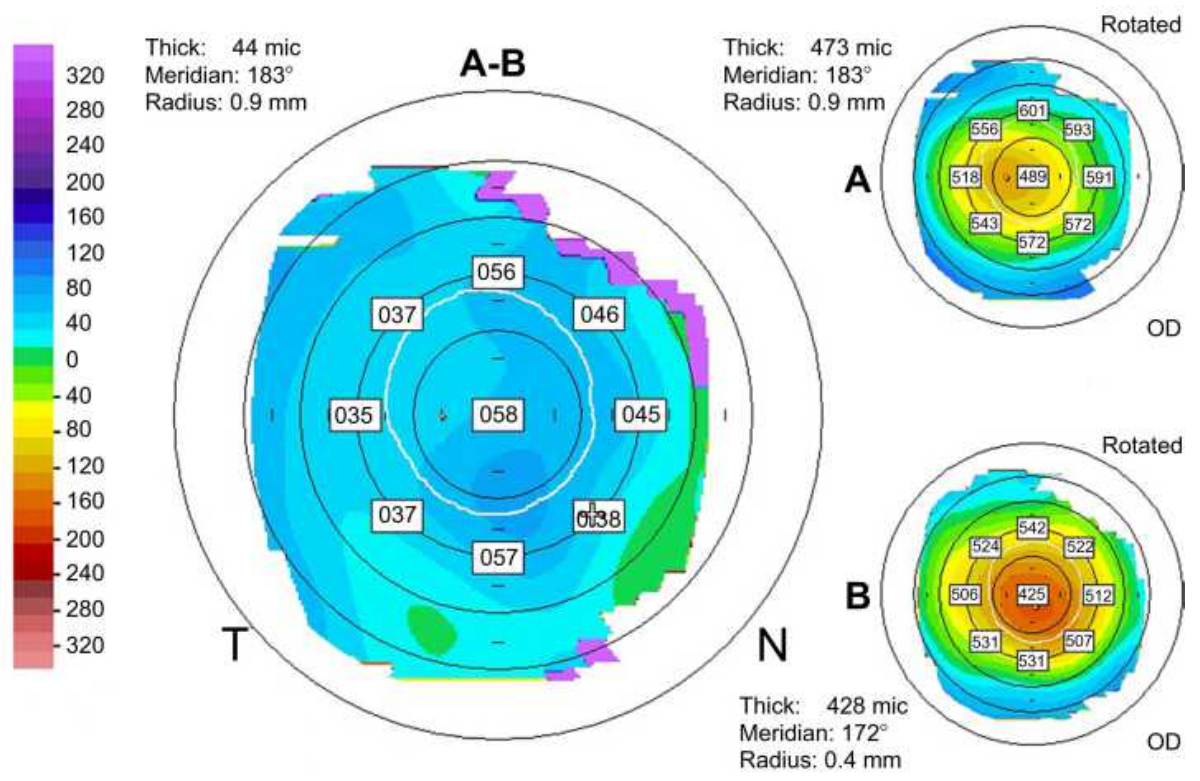

Figure 6 Differential pachymetric map by Orbscan II (Bausch \& Lomb, Bridgewater, NJ, USA) between pre- and post-E-LAK-SCAP in case I. 
Table I Patients Findings

\begin{tabular}{|l|l|l|l|l|}
\hline No of Case & I & $\mathbf{2}$ & $\mathbf{3}$ & $\mathbf{4}$ \\
\hline Male (M)/Female (F) & F & M & M & F \\
\hline Age (year) & & & & \\
\hline LRS & 23 & 26 & 19 & 46 \\
\hline E-LAK-SCAP & 32 & 31 & 20 & 48 \\
\hline Interval (months) from LRS to E-LAK-SCAP & 132 & 66 & 8 & 27 \\
\hline
\end{tabular}

Abbreviations: LRS, laser refractive surgery; E-LAK-SCAP, enhancement with laser epithelial keratomileusis-linked laser asymmetric keratectomy using semicylindrical ablation pattern.

Table 2 Intraoperative Findings

\begin{tabular}{|l|l|l|l|l|}
\hline No of Case & I & $\mathbf{2}$ & $\mathbf{3}$ & $\mathbf{4}$ \\
\hline Optic zone $(\mathrm{mm})$ & & & & \\
$\quad$ LRS & 6.2 & 6.0 & 6.0 & 6.0 \\
E-LAK-SCAP & 6.2 & 6.0 & 6.0 & 6.0 \\
\hline Ablation depth $(\mu \mathrm{m})$ & & & & \\
LRS & 48 & 63 & 78 & 90 \\
E-LAK -SCAP & 36 & 30 & 57 & 75 \\
\hline Residual stromal depth $(\mu \mathrm{m})$ & & & & \\
LRS & 420 & 395 & 410 & 391 \\
E-LAK-SCAP & 383 & 369 & 391 & 321 \\
\hline Myopic shift & & & & \\
Due to LAK-SCAP (diopters) & -1.50 & -1.75 & -2.25 & -2.50 \\
\hline
\end{tabular}

Abbreviations: LRS, laser refractive surgery; E-LAK-SCAP, enhancement with laser linked laser symmetric keratectomy using semi-cylindrical ablation pattern LAK-SCAP, laser symmetric keratectomy using semi-cylindrical ablation pattern.

LRS, $-1.0--3.75$ (average: -2.16 ) after LRS (ie, pre E-LAKSCAP), but $0--0.28$ (average: -0.16 ) after E-LAK-SCAP.

The UDVA (LogMAR) had increased after both surgeries (from an average of 1.00 to 0.65 after LRS, and from 0.65 to 0.00 after E-LAK-SCAP); hence, the E-LAKSCAP exhibited good outcomes for SE and UDVA. There were no cylindrical axial changes, and the CCT $(\mu \mathrm{m})$ had decreased after the two surgeries as well (from an average of 542 to 469 after LRS, and from 469 to 418 after E-LAK-SCAP). Further, the sum of the corneal thickness deviations in four directions (SUM) and the distance between the maximum posterior elevation (best-fit-sphere [BFS]) and the visual axis (DISTANCE) had increased after the LRS and decreased after the E-LAK-SCAP. The angle kappa values were similar before and after LRS, and lowered after the E-LAK-SCAP; the IOPs of the patients were similar before and after the LRS and LAK-SCAP. Moreover, the blurring scores had markedly decreased after the E-LAK-SCAP. Additionally, there was no myopic regression or blurring after the E-LAK-SCAP.

\section{Discussion}

In this study, in all four patients, SE and UDVA showed positive outcomes after LRS, but myopic regression of $-1.0--3.75$ diopters was present. After conventional LRS, when the SUM on an Orbscan map is $>80 \mu \mathrm{m}$, intraocular pressure causes the protrusion of regions of the cornea that have become thinner after the laser ablation easily, which steepens the cornea further, leading to myopic regression. ${ }^{2-4}$ Asymmetric thickness and curvature changes are explained by asymmetric biomechanical property distribution, and are difficult to explain otherwise. ${ }^{4}$ With LAK-SCAP, this parameter might be markedly decreased; in patients who underwent L-LAK-SCAP, the SUM was significantly decreased from 148 to $60 \mu \mathrm{m}$ (on an average) postoperatively. Moreover, the reduction in DISTANCE led to improvement in corneal symmetricity from an average of $1.25 \mathrm{~mm}$ to $0.36 \mathrm{~mm}$ after E-LAKSCAP. SCAP topography was assessed with an Orbscan map, and current Sheimpflug techniques were not used because only Orbscan map can calculate the distance between the BFS and visual axis, because there is a visual axis center map in Orbscan. This method was quite useful for measuring symmetry.

However, in two patients (Nos. 3 and 4). The SUM was markedly increased from 61.5 to $166.5 \mu \mathrm{m}$ after LRS due to decentered laser irradiations, and DISTANCE and corneal irregularities also increased in the $3.0 \mathrm{~mm}$ and $5.0 \mathrm{~mm}$ zones on the Orbscan map, but angle kappa remained similar. After E-LAK-SCAP, all patients showed symmetric cornea and SUM $<80 \mu \mathrm{m}$, decreased corneal irregularities on the Orbscan map, and increased UDVA. There was no myopic regression or blurring post E-LAKSCAP, which may be attributable to the excellent corneal point symmetry effect of E-LAK-SCAP. ${ }^{1-3}$ However, for reshaping of the cornea after E-LAK-SCAP and to fix the greater the $\mathrm{CCT}$, the higher postoperative time is required.

LAK-SCAP could be executed exactly with the help of Vision-Up software (WellC, South Korea) to analyze the corneal thickness deviations based on Orbscan II (Bausch \& Lomb, Bridgewater, NJ, USA) corneal pachymatric maps, and to predict the amounts of ablation in a thicker corneal region, the direction of axis (degree), number of SCAPs, and corneal myopic change as a result of the removal of the thicker corneal regions. Therefore, we 
Table 3 Pre- and Postoperative Findings of LRS and E-LAK-SCAP

\begin{tabular}{|c|c|c|c|c|c|c|c|c|c|c|c|c|}
\hline \multirow{2}{*}{$\begin{array}{l}\text { Items } \\
\text { Case }\end{array}$} & \multicolumn{4}{|c|}{ Pre LRS } & \multicolumn{4}{|c|}{ Pre E-LAK-SCAP (Post LRS) } & \multicolumn{4}{|c|}{ Post E-LAK-SCAP } \\
\hline & 1 & 2 & 3 & 4 & 1 & 2 & 3 & 4 & I & 2 & 3 & 4 \\
\hline SE (D) & -3.38 & -5.25 & -5.75 & -7.50 & -1.50 & -1.0 & -2.50 & -3.75 & 0 & -0.25 & -0.38 & 0 \\
\hline Sphere (D) & -2.75 & -5.25 & -5.0 & -6.50 & -1.50 & -1.0 & -2.0 & -3.25 & 0 & 0 & 0 & +0.50 \\
\hline Cylinder (D) & -1.25 & 0 & -1.50 & -1.0 & 0 & 0 & -1.0 & -1.0 & 0 & -0.50 & -0.75 & -1.0 \\
\hline IOP (mmHg) & 16 & 15 & 16 & 15 & 16 & 16 & 15 & 15 & 15 & 14 & 16 & 15 \\
\hline UDVA (LogMAR) & 1.00 & 1.00 & 1.00 & 1.00 & 0.50 & 0.10 & 1.00 & 1.00 & 0.00 & 0.00 & 0.00 & 0.00 \\
\hline Blurring score & - & - & - & - & 3 & 3 & 3 & 3 & 0 & 0 & 0 & 0 \\
\hline \multicolumn{13}{|l|}{$\mathrm{Cl}(\mathrm{D})$} \\
\hline 3.0-mm zone & I.I & 1.6 & 1.7 & 1.4 & 1.4 & 1.8 & 2.7 & 2.7 & 2.3 & 2.0 & 2.0 & 1.9 \\
\hline 5.0-mm zone & 1.5 & 1.5 & 1.7 & 1.5 & 1.6 & 2.0 & 2.6 & 2.8 & 4.0 & 2.1 & 2.2 & 2.2 \\
\hline CCT $(\mu \mathrm{m})$ & 540 & 533 & 555 & 541 & 479 & 463 & 514 & 420 & 427 & 402 & 473 & 369 \\
\hline $\operatorname{SUM}(\mu \mathrm{m})$ & 119 & 169 & 93 & 30 & 147 & 113 & 157 & 174 & 78 & 44 & 76 & 41 \\
\hline DISTANCE $(\mathrm{mm})$ & 0.57 & 1.02 & 1.02 & 0.71 & 1.32 & 1.22 & 1.63 & 0.81 & 0.54 & 0 & 0.77 & 0.14 \\
\hline Kappa angle $\left({ }^{\circ}\right)$ & 5.95 & 5.19 & 6.64 & 4.54 & 5.79 & 5.25 & 6.32 & 4.52 & 5.07 & 5.04 & 6.08 & 4.20 \\
\hline
\end{tabular}

Abbreviations: LRS, laser refractive surgery; E-LAK-SCAP, enhancement-linked laser asymmetric keratectomy using semi-cylindrical ablation pattern; D, diopters; IOP, intraocular pressure; LAK, laser asymmetric keratectomy; SE, spherical equivalent; UDVA, uncorrected distance visual acuity; LASIK, laser in-situ keratomileusis; LASEK, laser epithelial keratomileusis; $\mathrm{Cl}$, corneal irregularity (diopters) in 3.0-mm-and 5.0-mm zones on Orbscan map; CCT, central corneal thickness; SUM, sum of deviations in corneal thickness measurements in 4 directions based on Orbscan maps $(\mu \mathrm{m})$; DISTANCE, distance between the maximum posterior elevation (best fit sphere) and the visual axis.

were able to ablate the cornea to create central symmetry without changing refractive power. ${ }^{1-3}$

The pre- and one-year post-E-LAK-SCAP outcomes were also compared in this study. The one-year postoperative SUM was $<80 \mu \mathrm{m}$, which was superior to that of the pre- E-LAK-SCAP $(>80 \mu \mathrm{m})$; further, the post-E-LAKSCAP distance between the BFS and visual axis was $0.36 \mathrm{~mm}$ (average), which was superior to that of the preLRS (average, $1.25 \mathrm{~mm}$ ). Because LAK-SCAP is a recently developed technology, we were able to follow the patients up for one year in only four patients; thus, more studies with longer follow-up times, more than one year, and with a large number of patients are required in the future to investigate the outcomes, including myopic regression over time. The small sample size and short follow-up period are limitations of this study.

Using LAK-SCAP, the side effects caused by corneal deformity are expected to be significantly lower in cases with a SUM $(\geq 80 \mu \mathrm{m})$.

LAK has only been reported recently ${ }^{1-3}$ moreover, unlike the previously reported wavefront- and topography- guided LASIK or LASEK, LAK-SCAP decreases the sum of thickness deviations by asymmetric corneal ablation. Furthermore, it increases corneal symmetry by decreasing DISTANCE, and thereby, it is expected to prevent postoperative corneal changes. ${ }^{7-16}$ LAK-SCAP can asymmetrically ablate thick areas of the cornea compared to LASIK or LASEK, which symmetrically ablate the cornea. Additionally, it has been reported that LAK-SCAP, in which only the thick parts of the cornea are cut to create central symmetry, can be a good method to: 1) avoid LRS adverse effects, ${ }^{2,3}$ 2) treat the distorted cornea after cataract or glaucoma surgery, ${ }^{2,14,15} 3$ ) lessen the asymmetric morphology of the cornea and reduce the incidence of optical aberrations, ${ }^{1-14}$ and 4) reduce the effects of IOP pushing outward on the thin parts of the cornea in early keratoconus. ${ }^{17,18}$ In addition, further research on LAKSCAP and treatment indications needs to be conducted.

\section{Conclusion}

In conclusion, E-LAK-SCAP resulted in a decreased sum of deviations in corneal thickness and increased corneal 
symmetry with good visual outcomes one-year postoperatively.

\section{Acknowledgments}

We would like to thank Editage for English language editing.

\section{Disclosure}

The authors report no conflicts of interest in this work.

\section{References}

1. Agudo JAR, Park J, Park J, Lee S, Park K. Laser asymmetric corneal ablation to improve corneal shape. Lasers Med Sci. 2019;34 (9):1763-1779. doi:10.1007/s10103-019-02770-z

2. Min JS, Jin LY, Min BM. Early postoperative changes after laser asymmetric keratectomy for the management of adverse effects after photorefractive keratectomy. J Ophthalmol Open Access. 2020;1:1-7.

3. Min JS, Min BM. Comparison between surgical outcomes of LASIK with and without laser asymmetric keratectomy to avoid conventional laser refractive surgery adverse effects. Sci Rep. 2020;10(1):10446. doi:10.1038/s41598-020-67269-y

4. Roberts CJ, Dupps WJ Jr. Biomechanics of corneal ectasia and biomechanical treatments. J Cataract Refract Surg. 2014;40(6):991-998. doi:10.1016/j.jcrs.2014.04.013

5. Roberts CJ. Biomechanical customization: the next generation of laser refractive surgery. $J$ Cataract Refract Surg. 2005;31(1):2-5. doi:10.1016/j.jcrs.2004.11.032

6. Pop M, Payete Y. Risk factors for night vision complaints after LASIK for myopia. Ophthalmology. 2004;111(1):3-10. doi:10.1016/j. ophtha.2003.09.022

7. Ortiz D, Pinero D, Shabayek MH, Amalich-Montiel F, Alio JL. Corneal biomechanical properties in normal, post-laser in situ keratomileusis and keratoconic eyes. $J$ Cataract Refract Surg. 2007;33:1371-1375. doi:10.1016/j.jcrs.2007.04.021

8. Ambrosio R Jr, Nogueira LP, Caldas DL, et al. Evaluation of corneal shape and biomechanics before LASIK. Int Ophthalmol Clin. 2011;51 (2):11-38. doi:10.1097/IIO.0b013e31820f1d2d
9. Lee H, Roberts CJ, Kim TI, Ambrosio R Jr, Elsheikh A, Yong Kang DS. Change in biomechanically corrected intraocular pressure and dynamic corneal response parameters before and after transepithelial keratectomy and femtosecond laser-assisted laser in situ keratomileusis. $J$ Cataract Refract Surg. 2017;43:1495-1503. doi:10.1016/j.jcrs.2017.08.019

10. Osman IM, Halaly HY, Abdally M, Shousha MA. Corneal biomechanical changes in eyes with small incision lenticule extraction and laser assisted in situ keratomileusis. BMC Ophthalmol. 2016;16:123. doi:10.1186/s12886-016-0304-3

11. Wang B, Zhang Z, Naidu RK, et al. Comparison of the change in posterior corneal elevation and corneal biomechanical parameters after small incision lenticule extraction and femtosecond laser-assisted LASIK for high myopia correction. Cont Lens Anterior Eye. 2016;39(3):191-196. doi:10.1016/j.clae.2016.01.007

12. Wang D, Liu M, Chen Y, et al. Differences in the corneal biomechanical changes after SMILE and LASIK. J Refract Surg. 2014;30 (10):702-707. doi:10.3928/1081597X-20140903-09

13. Matalia J, Francis M, Gogri P, Panmand P, Matalia P, Sinha Roy A. Correlation of corneal biomechanical stiffness with refractive error and ocular biometry in a pediatric population. Cornea. 2017;36 (10):1221-1226. doi:10.1097/ICO.0000000000001290

14. Hirasawa K, Nakakura S, Nakao Y, et al. Change in corneal biomechanics and intraocular pressure following cataract surgery. $\mathrm{Am}$ J Ophthalmol. 2018;195:26-35. doi:10.1016/j.ajo.2018.07.025

15. Liu J, Roberts CJ. Influence of corneal biomechanical properties on intraocular pressure measurement: quantitative analysis. $J$ Cataract Refract Surg. 2005;31(1):146-155. doi:10.1016/j.jcrs.2004.09.031

16. Moshirfar M, Edmonds JN, Behunin NL, Christiansen SM. Corneal biomechanics in iatrogenic ectasia and keratoconus: a review of the literature. Oman J Ophthalmol. 2013;6:12-17. doi:10.4103/0974620X.111895

17. Wolffsohn JS, Safeen S, Shah S, Laiquzzaman M. Changes of corneal biomechanics with keratoconus. Cornea. 2012;31(8):849-854. doi:10.1097/ICO.0b013e318243e42d

18. Kerautret J, Colin J, Toubol D, Roberts C. Biomechanical characteristics of the ectatic cornea. J Cataract Refract Surg. 2008;34 (3):510-513. doi:10.1016/j.jcrs.2007.11.018
Clinical Ophthalmology

\section{Publish your work in this journal}

Clinical Ophthalmology is an international, peer-reviewed journal covering all subspecialties within ophthalmology. Key topics include: Optometry; Visual science; Pharmacology and drug therapy in eye diseases; Basic Sciences; Primary and Secondary eye care; Patient Safety and Quality of Care Improvements. This journal is indexed on PubMed
Central and CAS, and is the official journal of The Society of Clinical Ophthalmology (SCO). The manuscript management system is completely online and includes a very quick and fair peer-review system, which is all easy to use. Visit http://www.dovepress.com/ testimonials.php to read real quotes from published authors. 